\title{
First results from the ionospheric tomography experiment using beacon TEC data obtained by means of a network along a longitude of $136^{\circ} \mathrm{E}$ over Japan
}

\author{
Smitha V. Thampi and Mamoru Yamamoto \\ Research Institute for Sustainable Humanosphere (RISH), Kyoto University, Japan \\ (Received July 16, 2009; Revised September 7, 2009; Accepted October 17, 2009; Online published March 4, 2010)
}

\begin{abstract}
A chain of newly designed GNU (GNU is not UNIX) Radio Beacon Receivers (GRBR) has recently been established over Japan, primarily for tomographic imaging of the ionosphere over this region. Receivers installed at Shionomisaki $\left(33.45^{\circ} \mathrm{N}, 135.8^{\circ} \mathrm{E}\right)$, Shigaraki $\left(34.8^{\circ} \mathrm{N}, 136.1^{\circ} \mathrm{E}\right)$, and Fukui $\left(36^{\circ} \mathrm{N}, 136^{\circ} \mathrm{E}\right)$ continuously track low earth orbiting satellites (LEOS), mainly OSCAR, Cosmos, and FORMOSAT-3/COSMIC, to obtain simultaneous total electron content (TEC) data from these three locations, which are then used for the tomographic reconstruction of ionospheric electron densities. This is the first GRBR network established for TEC observations, and the first beacon-based tomographic imaging in Japanese longitudes. The first tomographic images revealed the temporal evolution with all of the major features in the ionospheric electron density distribution over Japan. A comparison of the tomographically reconstructed electron densities with the $f_{\mathrm{o}} F_{2}$ data from Kokubunji $\left(35^{\circ} \mathrm{N}\right.$, $\left.139^{\circ} \mathrm{E}\right)$ revealed that there was good agreement between the datasets. These first results show the potential of GRBR and its network for making continuous, unattended ionospheric TEC measurements and for tomographic imaging of the ionosphere.
\end{abstract}

Key words: Tomography, radio beacon, mid-latitude ionosphere, GNU radio beacon receiver.

\section{Introduction}

The use of beacons on low earth orbiting satellites (LEOS) emerged as a potential tool to investigate the twodimensional structure of the ionosphere soon after Austen et al. (1988) suggested that the tomographic technique using total electron content (TEC) data could be used for imaging the electron density distribution in the ionosphere. This technique has subsequently been widely used to study the large-scale structures of the ionosphere over high-, mid-, and low-latitude regions (e.g., see Raymund et al., 1993; Foster et al., 1994; Kunitsyn et al., 1995; Andreeva et al., 2000; Materassi et al., 2003; Thampi et al., 2007). The primary data used for the tomographic inversion is the line of sight TECs estimated along a number of ray paths from a chain of ground receivers aligned along the same longitude. The relationship between these data and the electron density can be described by

$$
\mathrm{TEC}=\int_{p} N(s) d s
$$

where $N(s)$ is the electron density distribution, and $p$ represents a ray path between the satellite and the ground receiver. The ground receivers basically measure the differential Doppler between the 150- and 400-MHz signals, both of which are transmitted coherently from a satellite-borne beacon, and these are related to the slant TEC along the ray. The TECs are estimated along a number of ray paths

Copyright (C) The Society of Geomagnetism and Earth, Planetary and Space Sciences (SGEPSS); The Seismological Society of Japan; The Volcanological Society of Japan; The Geodetic Society of Japan; The Japanese Society for Planetary Sciences; TERRAPUB.

doi:10.5047/eps.2009.10.003 that define the passage of a LEOS as determined by the ground-based receiver at a given location. Such observations from a chain of receivers (aligned along a meridian) are then inverted to obtain the electron density distribution as a function of latitude and altitude over a given longitude. A typical configuration used for ionospheric tomography, consisting of a LEOS and three ground-based receivers is shown in Thampi et al. (2004). The region to be imaged is divided into pixels, and the electron density in each pixel is assumed to be a constant. The ionospheric tomography problem can then be expressed mathematically by a set of simple linear algebraic equations, such as

$$
Y=A \boldsymbol{x}+E,
$$

where $Y$ is a vector of the observed TEC data, $\boldsymbol{x}$ is a vector of the unknown electron densities, and $A$ is the geometry matrix that describes the relationship between the line-ofsight TEC data and the electron densities. In other words, the elements of $A$ denote the length of a ray that passes through the pixels of interest. $E$ represents the measurement and discretization errors (Thampi et al., 2004). Since this is a highly ill-posed inverse problem, several iterative as well as non-iterative algorithms are currently being used for the inversion. A review of some of these methods is given by Raymund (1995). Unlike the single station measurements, tomographic studies can provide insight into the variability of the large-scale features of the ionosphere because of its better spatial coverage compared to single station measurements. The aim of this paper is to present the first experimental results from a newly installed network of beacon receivers over $136^{\circ} \mathrm{E}$ longitude sector in Japan and to show its potential to reveal the variability of the iono- 
sphere over the Japan region.

\section{Data and Algorithm}

A new digital receiver named the "GNU (GNU is not UNIX) Radio Beacon Receiver (GRBR)" was developed using the open-source hardware called Universal Software Radio Peripheral (USRP) and the open-source software toolkit for the software-defined radio (GNU Radio). The technical details of the receiver have been reported by Yamamoto (2008). The GRBR receivers were installed at Shionomisaki $\left(33.45^{\circ} \mathrm{N}, 135.8^{\circ} \mathrm{E}\right)$, Shigaraki $\left(34.8^{\circ} \mathrm{N}, 136.1^{\circ} \mathrm{E}\right)$, and Fukui $\left(36^{\circ} \mathrm{N}, 136^{\circ} \mathrm{E}\right)$ where they continuously track LEOS, mainly the OSCAR, Cosmos, RADCAL, DMSPF15, and FORMOSAT-3/COSMIC (F3/C) satellites. The OSCAR and Cosmos satellites are at an approximate altitude of $1000-1100 \mathrm{~km}$ in a nearly circular polar orbit, whereas the DMSP satellite is at an altitude of approximately $840 \mathrm{~km}$. The F3/C satellites are at an altitude of approximately $750-800 \mathrm{~km}$ with an orbital inclination of $72^{\circ}$. Consequently, the TECs between the ground receiver and LEOS are little affected by the plasmaspheric electron content.

The GRBR network has been operational since 10 July 2008. The receivers of this network continuously track the phase variation between the $150-$ and $400-\mathrm{MHz}$ signal, both of which are transmitted from these LEOS. The values of the differential phase between the 150- and 400$\mathrm{MHz}$ signals lie between 0 and $2 \pi$, and when we cumulate these phase values, it is possible to obtain continuous phase records with the minimum value of the curve arbitrarily set at zero. Hence, the relative TECs show zero value at the point of observation, where the line-of-sight distance is the minimum. The relative TEC measurements are converted to absolute TECs using the two-station method proposed by Leitinger (1975) and then used for the tomographic reconstruction. Here, we have used the algebraic reconstruction technique (ART) for generating the electron density values, which is the same approach as that used by many previous investigators (Materassi et al., 2003; Thampi et al., 2007). ART is a very simple iterative reconstruction technique, with a typical step

$$
x^{k+1}=x^{k}+\lambda_{k} \frac{y_{i}-\left\langle a^{i}, x^{k}\right\rangle}{\left\|a^{i}\right\|^{2}} a^{i}
$$

where $x^{k}$ represents the elements in the current image, $y_{i}$ is the TEC measurement along the $i$ th ray, $a_{i j}$ is its weight in the $j$ th pixel, and $\lambda_{k}$ represent the relaxation parameters. The solution converges in less than ten iterations. The region to be imaged is first divided into geocentric circular grids, each with a pixel resolution of $1^{\circ}$ (latitude) $\times$ $50 \mathrm{~km}$ (altitude), and the reconstruction is subsequently performed. The initial assumption necessary for the ART algorithm is obtained using the IRI-2007 model (Bilitza and Reinish, 2008). The reconstructions thus obtained are again interpolated and smoothed to obtain the images with a final resolution of $0.5^{\circ}$ (latitude) $\times 25 \mathrm{~km}$ (altitude). The region of reconstruction typically spans from $27^{\circ} \mathrm{N}$ to $42^{\circ} \mathrm{N}$ horizontally and from 100 to $650 \mathrm{~km}$ vertically. This wide spatial coverage with only three receiving stations is a major advantage of the tomographic technique over other groundbased measurements.

\section{Results and Discussion}

Figure $1(\mathrm{a}-\mathrm{h})$ shows the relative TEC data received at the three stations from different satellite passes on July 10 and 11,2008 . The $A_{\mathrm{p}}$ values on these days were 3 and 6 , respectively. The maximum elevations were $\geq 70^{\circ}$ for all passes, except those shown in Fig. 1(b-d) which had maximum elevation angles of between 40 and $50^{\circ}$ at different locations. Figure 1(a) corresponds to a COSMOS2407 pass from south to north (referred to as the SN pass hereafter) at $\sim 1151$ UT (2051 LT) on July 10, 2008. Small perturbations can be clearly seen in the relative TEC data; these are typical of the mid-latitude summer nighttime over Japan (Saito et al., 1998). In contrast, in the next pass of COSMOS 2407 (from north to south, referred to as NS pass) at $\sim 2251$ UT (0751 LT on the next day, the relative TECs are more smooth (Fig. 1(b)), which corresponds to a typical morning time situation. The equatorial ionization anomaly (EIA) crests are expected to be weaker and closer to the equator at these local times (Sastri, 1990). Figure 1(c) shows the data from an OSCAR $32 \mathrm{SN}$ pass at 0618 UT (1518 LT). From relative TECs shown in this figure, it is evident that the latitudinal gradient is much higher on the southern side than in the northern region. This result is to be expected in the daytime because of the higher electron densities associated with the EIA feature in the lowlatitude region. Similarly, Fig. 1(d) shows the data from an OSCAR $32 \mathrm{SN}$ pass at $0806 \mathrm{UT}$ (1706 LT). At this time also, the latitudinal gradient is higher on the southern side than in the northern region because of the presence of EIA. The following datasets were obtained at 0958 UT $(1858$ LT) (Fig. 1(e)). Figure 1(f) shows the data obtained at 1217 UT (2117 LT), which corresponds to a SN pass of COSMOS 2407. Signatures of wave-like perturbations on the northern side are much stronger at this time than during the previous night. Another interesting feature of Fig. 1(f) is that the relative TEC gradient on the northern side seems to be higher than that on the southern side, which is in contrast to the daytime condition. This feature is persistent in the next satellite pass at 1959 UT (0459 LT, next day) as well (Fig. 1(g)). This was a SN pass of the FM6 satellite of the $\mathrm{F} 3 / \mathrm{C}$ constellation. The relative TEC in the southern region can be seen to be much lower than that in the northern region. However, the signatures of the travelling ionospheric disturbances (TIDs) are absent in this data, indicating that the medium scale TID (MSTID) activity has ceased by this time. The next dataset at 2157 UT (0657 LT, next day) shows that the relative TECs in both the southern and northern regions have enhanced after sunrise (Fig. 1(h)). Based on these data, it can be seen that the relative TEC observations themselves can provide significant information on not only the latitudinal distribution of the ionosphere at different local times but also on the presence of features like MSTIDs. These TEC observations can then be used to generate the tomographic images of electron density.

Figure 2(a-h) show the series of tomographic images obtained using the data shown in Fig. 1 $(\mathrm{a}-\mathrm{h})$. These images represent the temporal evolution of the ionosphere over this 


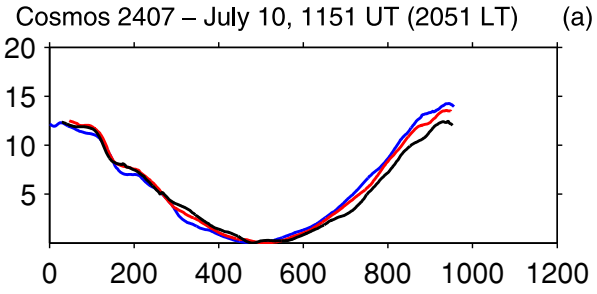

Cosmos 2407 - July 10, 2251 UT (July 11, 0751 LT) (b)
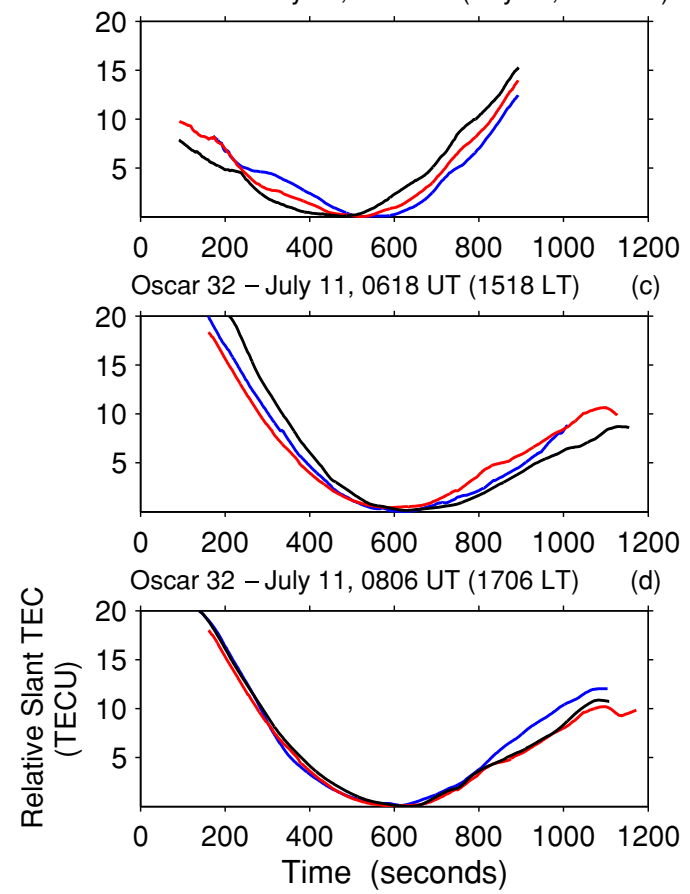

Oscar 25 - July 11, 0958 UT (1858 LT)

(e)

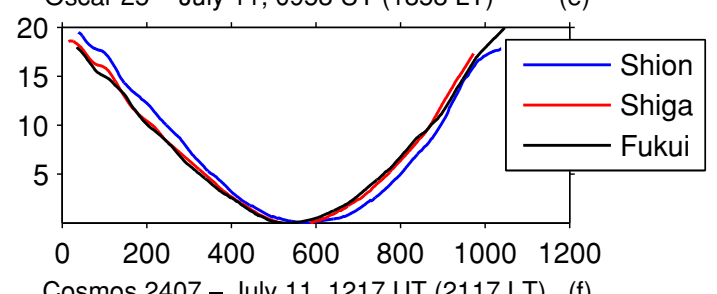

Cosmos 2407 - July 11, 1217 UT (2117 LT) (f)
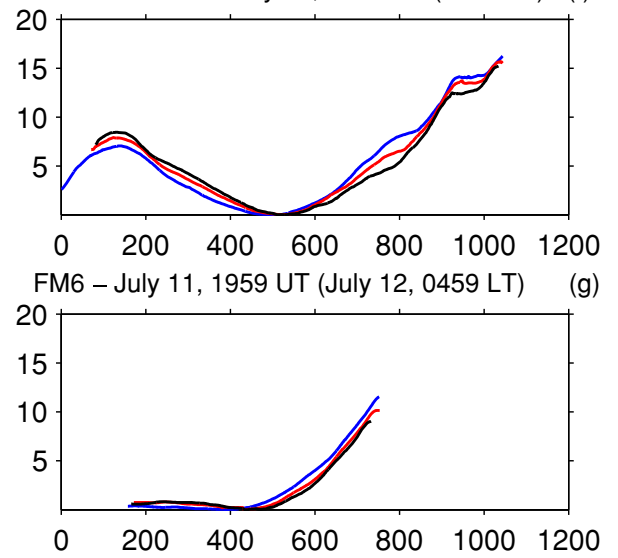

Oscar 25 - July 11, 2157 UT (July 12, 0657 LT) (h)

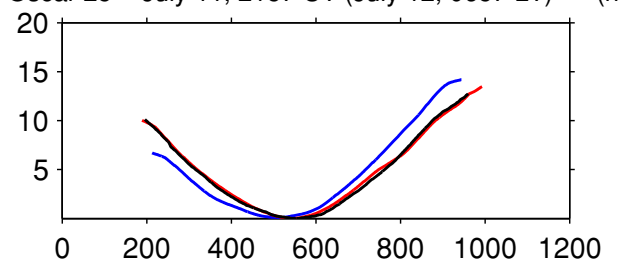

Fig. 1. The temporal variation of TEC data obtained from different satellite passes from the GRBR network stations on July 10 and 11,2008 .

region from July 10, 1200 UT (2100 LT) to July 11, 22 UT (0700 LT, next day). Figure 2(a) shows the tomographic image obtained using the data from the three stations as shown in Fig. 1(a). The image gives some indication of the presence of perturbations in the electron density distribution. Another interesting feature that can be observed in Fig. 2(a) is that the densities in the northern latitudes are higher than those over the southern latitudes. This is the signature of the mid-latitude summer nighttime anomaly (MSNA) reported by Lin et al. (2009). The evening time enhancement of TEC in the summer season over Japan has also reported by Maruyama (2007) using GPS TEC measurements. The MSNA can be described as a state in which the nighttime plasma density at the mid-latitude region exceeds the daytime values, and the density at the northern mid-latitudes $\left(>\sim 33-34^{\circ} \mathrm{N}\right)$ often remains higher than that at latitudes of less than $\sim 33^{\circ} \mathrm{N}$ in the nighttime. This feature can also be observed on the images obtained on the next day (Fig. 2(f, g)). More detailed results on the features of MSNA, based on a variety of experiments including tomography, have been reported by Thampi et al. (2009).

Figure 2(b) shows the reconstruction using the data shown in Fig. 1(b). The densities are now much higher than in the previous case, and the electron densities in the lower altitudes $(100-150 \mathrm{~km})$ are higher than those obtained in the nighttime. The enhanced electron densities in the northern region have disappeared, and there is now greater elec- tron density on the southern side than in the northern region, which is to be expected at this local time (0800 LT). The tomographic images shown in Fig. 2(c, d) correspond to the data obtained from the subsequent passes at $0618 \mathrm{UT}$ (1518 LT) and 0806 UT (1706 LT), respectively. These images show a clear enhancement in electron densities in the southern region relative to the northern region. The maximum electron density in the southern region is observed in the image obtained at these times, which agrees with the daytime EIA behavior in the low-latitude region (Sastri, 1990). The EIA feature has been successfully imaged using tomographic methods (e.g., Andreeva et al., 2000; Franke et al., 2003; Materassi et al., 2003; Yizengaw et al., 2007). It must be mentioned here that what we are seeing is not the crest of the EIA (which would be further south of the imaged region), but the edge of the EIA-associated enhancement, because of which the southern region has more electron density than the northern region. It can also be seen that unlike the nighttime, the electron density structure is rather smooth without any major perturbations.

Figure 2(e) shows the tomographic image at 0958 UT (1858 LT). Here, the large electron densities in the southern latitudes have started to disappear. The maximum density associated with the EIA is found at 1400-1500 LT, which agrees with previous observations (e.g., Lin et al., 2007). The reconstruction corresponding to the next satellite pass at 1217 UT (2117 LT) shows that the northern side has en- 

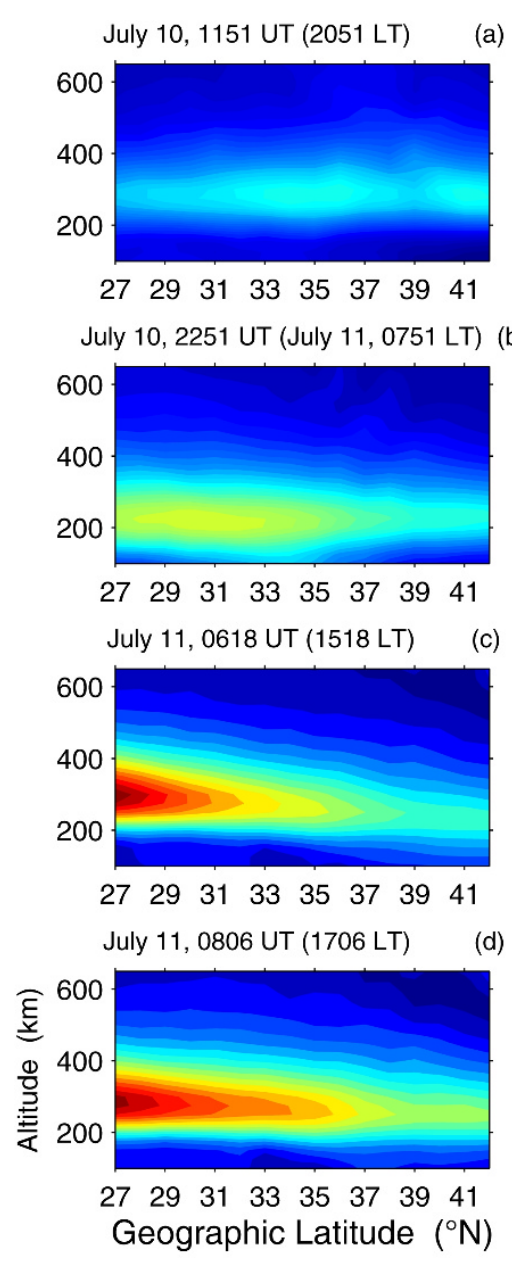
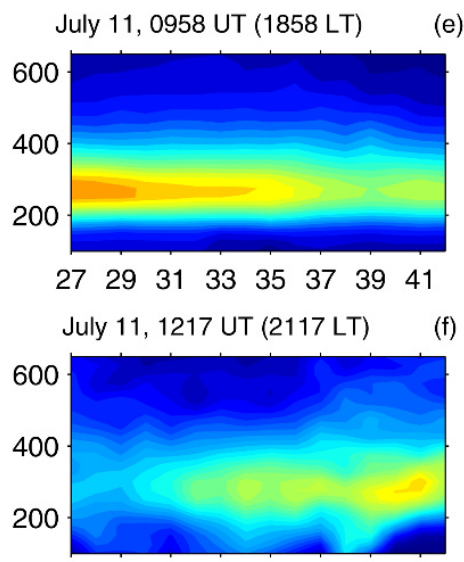

$\begin{array}{llllllll}27 & 29 & 31 & 33 & 35 & 37 & 39 & 41\end{array}$

July 11, 1959 UT (July 12, 0459 LT) (g)

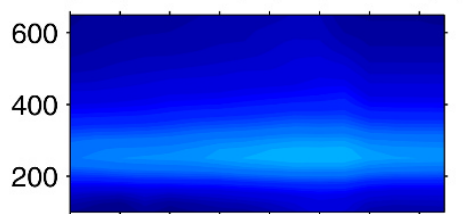

$\begin{array}{llllllll}27 & 29 & 31 & 33 & 35 & 37 & 39 & 41\end{array}$

July 11, 2157 UT (July 12, 0657 LT)

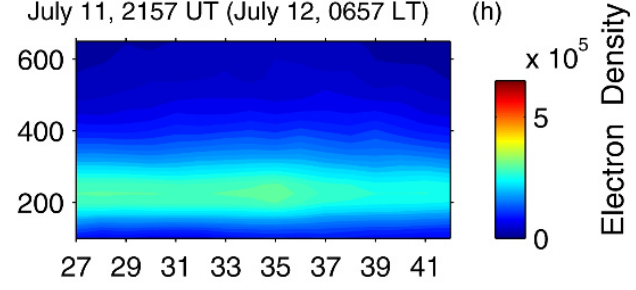

Fig. 2. The series of tomographic images obtained using the data shown in Fig. 1(a-h).

hanced densities compared to the southern side; the presence of the MSTID feature is also evident (Fig. 2(f)), especially in the northern latitudes. Tomographic methods have been successfully used in past studies to investigate the spatial structure of TIDs over other longitude sectors (Cook and Close, 1995; Pryse et al., 1995; Yizengaw et al., 2005). The typical horizontal wavelength of $\sim 150-200 \mathrm{~km}$ (in the NS direction) can be roughly identified from this image. Nonetheless, the estimation of the wavelength of MSTIDs using the tomographic technique is limited by the horizontal resolution of the reconstruction. A comparison of Fig. 2(a) and 2(f) shows that the electron densities on July 11 were slightly higher than those on the previous day, even with the same initial assumption applied in the reconstruction for both the days and a stronger MSNA on July 11 than on July 10 . Another interesting feature is that in the latitude region $>35^{\circ} \mathrm{N}$, the nighttime density exceeds the daytime values, which is another characteristic of MSNA (Thampi et al., 2009). Figure 2(g) shows the image after approximately $8 \mathrm{~h}$, and it can be seen that the MSTID structure has disappeared completely and that the overall electron density has reached the minimum, which was quite anticipated. The last image of the series of tomograms (Fig. 2(h)) was obtained at 2157 UT (0657 LT, July 12) and shows the typical morning-time ionosphere, with higher densities than in the previous image. This is a typical picture of the early

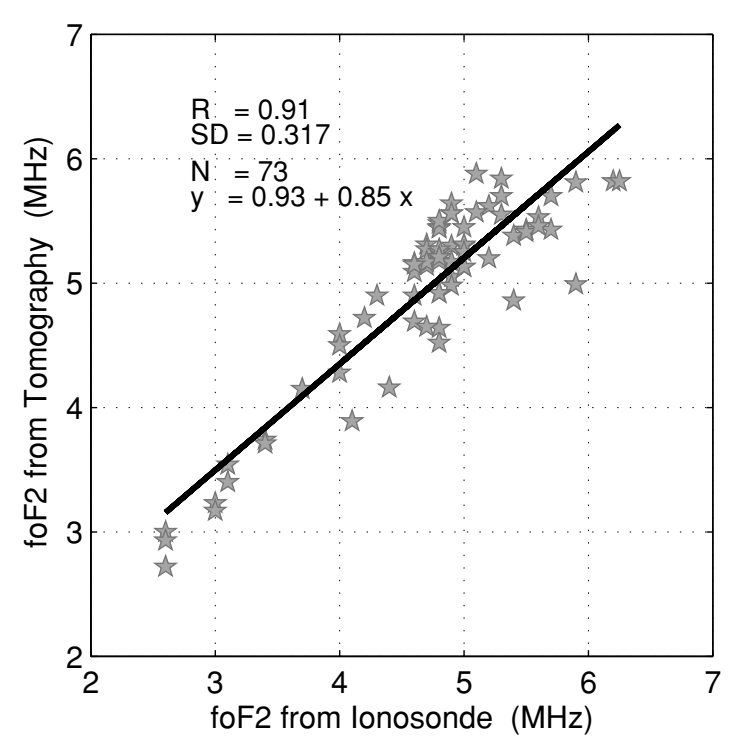

Fig. 3. The comparison of Nmax values derived from tomographic images and ionosonde. The solid line shows the linear best fit.

morning mid-latitude ionosphere.

The maximum electron density $\left(N_{\max }\right)$ values obtained from the tomographic reconstructions were compared with those derived from the ionosonde located at Kokubunji 
$\left(35^{\circ} \mathrm{N}, 139^{\circ} \mathrm{E}\right)$ (Fig. 3). In generating the tomographic images, we did not use the bottomside profile from the ionosonde as the initial profile and, therefore, the images reconstructed using TEC data are completely independent of the ionosonde data. A few cases in which the solution of the inversion did not converge within a difference of $40 \%$ in $f_{\mathrm{o}} F_{2}$, when compared with the ionosonde data were totally discarded as 'unreliable' reconstructions. This often occurs due to limitations in the data, either in quality or coverage. After discarding such images, we obtained a total of 73 images, which were used further. The correlation was found to be $\sim 0.9$ and was significant at $>95 \%$. It must be realized that one cannot expect an exact agreement between the maximum electron density and the ionosonde signals because the peak density from tomograms is averaged over a pixel of vertical size $50 \mathrm{~km}$, whereas the ionosonde signals may be scattered from a particular height.

The $h_{\mathrm{m}} F_{2}$ values can be determined from the ionosonde observations using a simple relation given by Bradley and Dudeney (1973).

$$
h_{\mathrm{m}} F_{2}=\frac{1490}{M(3000) F_{2}+\Delta M}-176
$$

Where,

$$
\Delta M=\frac{0.18}{\frac{f_{\mathrm{o}} F_{2}}{f_{\mathrm{o}} E}-1.4}
$$

A comparison of such estimates with the tomographic observations yielded a correlation with $R=0.3$ for the entire dataset, and a slightly better correlation with $R=0.5$ when the nighttime values alone were considered for comparison (not illustrated). The discrepancy between these estimate arises from two factors: (1) the tomography gives the $h_{\mathrm{m}} F_{2}$ with a resolution of $25 \mathrm{~km}$, whereas the ionosonde estimates are 'point measurements'; (2) the estimate of $h_{\mathrm{m}} F_{2}$ using Eq. (4) is only an approximation to the actual $h_{\mathrm{m}} F_{2}$, especially when the values of $f_{\mathrm{o}} E$ are not reliable. Similarly, the tomographic estimates of $h_{\mathrm{m}} F_{2}$ largely depend on the $h_{\mathrm{m}} F_{2}$ value given as the initial guess. In short, both estimates are limited in terms of accuracy. It must also be noted here that Kokubunji is about $\sim 300 \mathrm{~km}$ away from the chain and, horizontally, this is greater than the wavelength of MSTIDs. Depending on the azimuth and elevation angle of the satellite pass, the distance between the satellite observation and Kokubunji would vary. These are the limitations inherent in doing such comparisons. Nevertheless, these comparisons serve as an easy tool to validate the tomographic reconstructions.

\section{Conclusions}

The first ground-based GRBR network established for tomography experiments is now being used for the first time to monitor the ionospheric TEC variability. Beacon tomographic imaging of the ionosphere is being performed for the first time from Japanese longitudes. Here, we present the initial results from the tomography experiments. The images reveal the temporal evolution as well as prominent features of the mid-latitude ionosphere, such as TIDs and MSNA. A comparison with the $f_{\mathrm{o}} F_{2}$ values obtained from ionosonde data were used to validate the tomographic reconstruction. Selected examples of tomographic images shown here illustrate that tomographic reconstruction can reveal the temporal evolution and variability of the midlatitude ionosphere. Data from the GRBR network are currently being used to study the structures of the ionosphere around Japan. The GRBR and its network have proven to be quite useful for the continuous, unattended ionospheric TEC measurements and for the tomographic imaging of the ionosphere. The results also reiterate the usefulness of theGRBR network for other regions as well, especially for the routine observations and the potential of tomographic imaging to provide information on ionospheric variability over a wide spatial region.

Acknowledgments. The work of ST is supported by the Japan Society for the Promotion of Science (JSPS) foundation. The authors thank Dr. Akinori Saito for his support in the installation of the GRBR network, and NICT, Japan, for the ionosonde data.

\section{References}

Andreeva, E. S., S. J. Franke, K. C. Yeh, and V. E. Kunitsyn, Some features of the equatorial anomaly revealed by ionospheric tomography, Geophys. Res. Lett., 27, 2465-2468, 2000.

Austen, J. R., S. J. Franke, and C. H. Liu, Ionospheric imaging using computerized tomography, Radio Sci., 23, 299-307, 1988.

Bilitza, D. and B. W. Reinish, International Reference Ionosphere 2007: Improvements and new parameters, Adv. Space Res., 42, 599-609, 2008.

Bradley, P. A. and J. R. Dudeney, A simple model of the vertical distribution of electron concentration in the ionosphere, J. Atmos. Terr. Phys., 35(12), 2131-2146, 1973.

Cook, J. A. and S. Close, An investigation of TID evolution observed in MACE '93 data, Ann. Geophys., 13, 1320-1324, 1995.

Foster, J. C., J. A. Klobuchar, V. E. Kunitsyn, E. D. Tereshchenkov, E. S. Andreeva, M. J. Bounsanto, P. Fougere, J. M. Holt, B. Z. Khudukon, W. Pakula, and T. D. Raymund, Russian American tomography experiment, Int. J. Imaging Syst. Techn., 5, 148-159, 1994.

Franke, S. J., K. C. Yeh, E. S. Andreeva, and V. E. Kunitsyn, A study of the equatorial anomaly ionosphere using tomographic images, Radio Sci., 38(1), 1011, doi:10.1029/2002RS002657, 2003.

Kunitsyn, V. E., E. D. Tereshchenko, E. S. Andreeva, B. Z. Khudukon, and Y. A. Melnichenko, Radiotomographic investigations of ionospheric structures at auroral and middle latitudes, Ann. Geophys., 13, 12421253, 1995.

Leitinger, R., G. Schmidt, and A. Tauriainen, An evaluation method combining the differential Doppler measurements fromm two stations that enables the calculation of electron content of the ionosphere, J. Geophys., 41, 201-213, 1975.

Lin, C. H., J. Y. Liu, T. W. Fang, P. Y. Chang, H. F. Tsai, C. H. Chen, and C. C. Hsiao, Motions of the equatorial ionization anomaly crests imaged by FORMOSAT-3/COSMIC, Geophys. Res. Lett., 34, L19101, doi:10.1029/2007GL030741, 2007.

Lin, C. H., J. Y. Liu, C. Z. Cheng, C. H. Chen, C. H. Liu, W. Wang, A. G. Burns, and J. Lei, Three-dimensional ionospheric electron density structure of the Weddell Sea Anomaly, J. Geophys. Res., 114, A02312, doi:10.1029/2008JA013455, 2009.

Maruyama, T., Regional reference total electron content model over Japan based on neural network mapping techniques, Ann. Geophys., 25, 26092614, 2007.

Materassi, M., C. N. Mitchell, and P. S. J. Spencer, Ionospheric imaging of the northern crest of the equatorial anomaly, J. Atmos. Terr. Phys., 65, 1393-1400, 2003.

Pryse, S. E., C. N. Mitchell, J. A. T. Heaton, and L. Kersley, Tomographic imaging of travelling ionospheric disturbances, Ann. Geophys., 13, 1325-1331, 1995.

Raymund, T. D., Comparison of several ionospheric tomography algorithms, Ann. Geophys., 13, 1254-1262, 1995.

Raymund, T. D., S. E. Pryse, J. A. T. Heaton, and L. Kersley, Tomographic reconstruction of ionospheric electron density with European incoherent scatter radar verification, Radio Sci., 28, 811-817, 1993.

Saito, A., S. Fukao, and S. Miyazaki, High resolution mapping of TEC 
perturbations with the GSI GPS network over Japan, Geophys. Res. Lett., 25, 3079-3082, 1998.

Sastri, J. H., Equatorial anomaly in F region-A review, Indian J. Radio. Space Phys., 19, 225-240, 1990.

Thampi, S. V., T. K. Pant, S. Ravindran, C. V. Devasia, and R. Sridharan, Simulation studies on the tomographic reconstruction of the equatorial and low latitude ionosphere in the context of the Indian tomography experiment,-CRABEX, Ann. Geophys., 22, 3445-3460, 2004.

Thampi, S. V., S. Ravindran, C. V. Devasia, P. Sreelatha, T. K. Pant, R. Sridharan, Venkata Ratnam, A. D. Sharma, C. Raghava Reddi, J. Jose, and J. H. Sastry, Coherent radio beacon experiment (CRABEX) for tomographic imaging of the equatorial ionosphere in the Indian longitudes-Preliminary results, Adv. Space Res., 40, 436-441, 2007.

Thampi, S. V., C. Lin, H. Liu, and M. Yamamoto, First tomographic observations of the Midlatitude Summer Nighttime Anomaly over Japan,
J. Geophys. Res., 114, A10318, doi:10.1029/2009JA014439, 2009. Yamamoto, M., Digital beacon receiver for ionospheric TEC measurement developed with GNU Radio, Earth Planets Space, 60, e21-e24, 2008. Yizengaw, E., P. L. Dyson, E. A. Essex, and M. B. Moldwin, Ionosphere dynamics over the Southern Hemisphere during the 31 March 2001 severe magnetic storm using multi-instrument measurement data, Ann. Geophys., 23, 707-721, 2005.

Yizengaw, E., M. B. Moldwin, P. L. Dyson, and E. A. Essex, Using tomography of GPS TEC to routinely determine ionospheric average electron density profiles, J. Atmos. Sol. Terr. Phys., 69(3), 314-32, 2007.

S. V. Thampi (e-mail: smithathampi.rish@gmail.com) and M. Yamamoto 